\title{
Estudios Cariotípicos en Flourensia (Asteraceae) de Argentina
}

\author{
NATALIA DELBÓN ${ }^{1,2}$, GABRIEL BERNARDELLO ${ }^{1}$, MARÍA TERESA COSA ${ }^{1}$ y \\ LAURA STIEFKENS ${ }^{1}$
}

\begin{abstract}
Summary: Karyotypic studies in Flourensia (Asteraceae) from Argentina. Twelve species of the American genus Flourensia DC. inhabit Argentina. Six of them grow in its central region, all endemic to it. Chromosomal number is known for most species, but no karyotypic studies have been done. Therefore, we studied the mitotic chromosomes of the six Central Argentinean species, performing qualitative and quantitative analysis of their karyotypes. Permanent mounts were made from root tips with classical techniques. All species had $2 n=36$ : three with $14 m+4 s m$ pairs and the remaining with $12 m+6 s m$ pairs. The average chromosome length varied between 2.11 and $2.97 \mu \mathrm{m}$. Statistical differences were found for the variables $C, L t, R$, and $A_{1}$. Except for $F$. campestris, one or two pairs of satellites which varied in their location were found. The data obtained have taxonomic value since differences were found among nearly all species, suggesting that their speciation would have been accompanied by small chromosomal changes caused by inversions and/or translocations.
\end{abstract}

Key words: Central Argentina, Asteraceae, karyotype, cytogenetics, Flourensia, chromosome number.

Resumen: En Argentina crecen 12 especies del género americano Flourensia DC. En su zona central habitan seis, todas endémicas de dicha región. Se conoce el número cromosómico de la mayoría de las especies, pero no hay estudios cariotípicos. Por ello, se estudiaron los cromosomas mitóticos de las seis especies centro-argentinas, realizando un análisis cualitativo y cuantitativo de sus cariotipos. Se realizaron preparados permanentes de ápices radicales con técnicas clásicas. Todas las especies presentaron $2 n=36$ : tres de ellas con 14 pares $m+4 \mathrm{sm}$ y las restantes con 12 pares $m+6 \mathrm{sm}$. La longitud cromósomica promedio varió entre 2,11 y 2,97 $\mu \mathrm{m}$, habiéndose encontrado diferencias estadísticas en las variables largo cromosómico promedio, largo total del genoma haploide, índice braquial promedio y en el índice de asimetría intracromosómica. En todas las especies, excepto en $F$. campestris, se encontró uno o dos pares de satélites que variaron en su ubicación. Los datos obtenidos tienen valor taxonómico ya que se han encontrado diferencias entre casi todas las especies; además, sugieren que su especiación habría sido acompañada por pequeños cambios cromosómicos causados por inversiones y/o translocaciones.

Palabras clave: Argentina central, Asteraceae, cariotipo, citogenética, Flourensia, número cromosómico.

\section{INTRODUCCIÓN}

Asteraceae es una de las familias más dominantes del planeta ya que comprende ca. 1.700 géneros y entre 23.000-26.000 spp. distribuidas por todo el mundo, excepto en la Antártida (Funk et al., 2009). En Argentina, es la más numerosa con ca.

1 Instituto Multidisciplinario de Biología Vegetal (Universidad Nacional de Córdoba-CONICET), Casilla de Correo 495, 5000 Córdoba.

${ }^{2}$ Autor correspondiente: natalia_delbon@hotmail.com
1.400 spp., siendo endémicas 382 (Katinas et al., 2007). Su diversificación y gran expansión se debe a su capacidad adaptativa a diferentes climas, suelos y relieves, su eficacia reproductiva y sus estrategias para la dispersión y germinación de las semillas (Katinas et al., 2007). Muchas especies son económicamente importantes para el hombre, como alimentos, bebidas, medicinas, insecticidas, aceites, especias, edulcorantes, etc. (Simpson, 2009).

Flourensia DC. (subfam. Asteroideae, tribu Heliantheae) es endémico de América con 32 spp. (Dillon, 1984; Ariza Espinar, 2000). Heliantheae se compone de 113 géneros y ca. 1.641 spp. y es una de 
las más importantes de la 13 tribus que componen la Alianza Heliantheae, siendo considerada una de las más derivadas de la familia, junto con Millerieae, Perityleae, Eupatorieae y Madieae (Panero, 2007a, b; Baldwin, 2009). Estudios moleculares recientes agrupan a Encelia Adans., Enceliopsis A. Nelson, Flourensia, Geraea Torr. \& A. Gray y Helianthella Torr. \& A. Gray dentro de la subtribu Encellinae (Panero et al., 1999; Panero, 2005; Panero, 2007b; Fehlberg \& Ranker, 2007).

En los últimos años, las especies de Flourensia han cobrado importancia ya que sus resinas poseen potencial valor económico; por ello, hay numerosos trabajos que caracterizan sus compuestos químicos y determinan sus posibles usos (e.g., Uriburu et al., 2004; Vaca Ruiz et al., 2006; Joray et al., 2011).

En Argentina crecen 12 spp., de las cuales en su zona central habitan seis, todas endémicas de dicha región y con distribución restringida: $F$. campestris Griseb., F. hirta S. F. Blake, F. leptopoda S. F. Blake, F. niederleinii S. F. Blake, F. oolepis S. F. Blake y F. tortuosa Griseb. (Dillon, 1984; Ariza Espinar, 2000). Crecen en zonas serranas, en ambientes de clima semiárido y suelos empobrecidos. Se trata de arbustos con capítulos radiados de abundantes y vistosas flores amarillas, con un característico aspecto lustroso debido al exudado resinoso de sus hojas y ramas (Ariza Espinar, 2000). Son reconocidas en medicina popular para aliviar enfermedades gastrointestinales, como purgantes, expectorantes y antirreumáticas; algunas son usadas como incienso, aromáticas y tintóreas (e.g. Zardini, 1984; Barboza et al., 2006; Trillo et al., 2007).

Las características cariotípicas han probado ser importantes en estudios sistemáticos y evolutivos en muchos grupos de angiospermas (e.g., Shan et al. 2003; Urdampilleta et al. 2005), incluyendo las Asteraceae (e.g., Vanzela et al., 2002; WeissSchneeweiss et al., 2009; Moreno et al., 2012). En Flourensia son escasos los estudios citológicos, si bien se conoce el número cromosómico de 24 especies, siendo $\mathrm{n}=18 \mathrm{o}$ ca. 18 (Turner \& Johnston, 1961; Dillon, 1979, 1981, 1984, 1986; Di Fulvio, 1977; Weedin \& Powell, 1980; Robinson et al., 1981; Molau, 1986; Strother, 1983; Powell, 1984; Waisman et al., 1986; Wulff et al., 1996; Carr et al., 1999). Sobre estos antecedentes, el objetivo general de este trabajo fue estudiar los cromosomas mitóticos de seis especies centro-argentinas de Flourensia, realizando un análisis cualitativo y cuantitativo de sus cariotipos, con el fin de aportar datos que permitan una mayor comprensión de sus relaciones sistemáticas y filogenéticas.

\section{Materiales y Métodos}

Los análisis cariológicos se efectuaron en semillas de poblaciones Argentinas (Tabla 1), los ejemplares de herbario se encuentran depositados en CORD.

Las semillas se lixiviaron durante 24 horas con agua corriente y se colocaron en cajas de Petri con

Tabla 1. Poblaciones estudiadas de Flourensia, los datos de colección incluyen provincia,

departamento, lugar, georeferencia, altura sobre el nivel del mar, colector, número de muestra y fecha

$$
\text { de recolección. }
$$

\begin{tabular}{|c|c|}
\hline Especie & Datos de recolección \\
\hline F. campestris & $\begin{array}{l}\text { Prov. Córdoba: Dpto. Punilla, Cerro El Cuadrado, La Falda, S } 31^{\circ} 0,5^{\prime}-\mathrm{W} 64^{\circ} 27^{\prime}, 1100 \text { m s.n.m., } 21- \\
\text { III-2010, Delbón } 7 .\end{array}$ \\
\hline F. hirta & $\begin{array}{l}\text { Pov. La Rioja: Dpto. Famatina, Campana, S } 28^{\circ} 33^{\prime}-\mathrm{W} 67^{\circ} 37^{\prime} \text {, } 1640 \text { m s.n.m., 20-III-2010, Barboza } \\
2460 .\end{array}$ \\
\hline F. leptopoda & $\begin{array}{l}\text { Prov. La Rioja: Dpto. General San Martín, Ulapes, Sierra de Ulapes, S } 31^{\circ} 34^{\prime} \text {-W } 66^{\circ} 15^{\prime}, 695 \text { m s.n.m., } \\
\text { 18-III-2010, Barboza } 2438 .\end{array}$ \\
\hline F. niederleinii & $\begin{array}{l}\text { Prov. La Rioja: Dpto. Sanagasta, Las Peñas, S } 29^{\circ} 11^{\prime}-\mathrm{W} 67^{\circ} 3^{\prime}, 1200 \text { m s.n.m., 19-III-2010, Barboza } \\
2450 .\end{array}$ \\
\hline F. oolepis & $\begin{array}{l}\text { Prov. Córdoba: Dpto. Punilla, Capilla del Monte, Dique El Cajón, S 3051'-W 64³3', } 970 \text { m s.n.m., } \\
\text { 21-III-2010, Delbón } 8 .\end{array}$ \\
\hline F. tortuosa & 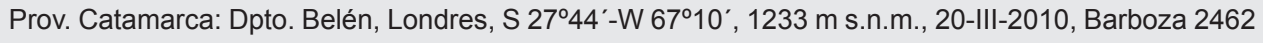 \\
\hline
\end{tabular}




\section{N. Delbón et al. - Estudios cariotípicos en Flourensia}

papel de filtro húmedo. Se las mantuvo en cámara con ambiente controlado con $25^{\circ} \mathrm{C}, 12$ horas de luz y 12 horas de oscuridad, regándolas diariamente con agua destilada. Los ápices radicales fueron cortados cuando las raíces tuvieron 5-10 mm. Se realizó un pretratamiento con una solución saturada de paradicloro-benceno durante 3 horas a temperatura ambiente (Mayer, 1945), luego se lavaron las raíces con agua destilada y se las fijó en una mezcla 3:1 de alcohol etílico absoluto:ácido acético glacial, preparada en el momento de usar, durante 24 horas a temperatura ambiente.

Se realizó la coloración de Feulgen (Jong, 1997) con el siguiente procedimiento: lavado de las raíces con agua destilada 5-10 minutos, hidrólisis en ácido clorhídrico $5 \mathrm{~N}, 40$ minutos a temperatura ambiente, lavado con agua destilada 5 minutos, secado de raíces en papel de filtro, coloración con Reactivo de Schiff en oscuridad 2 horas a temperatura ambiente y realización del preparado en un portaobjetos con una gota de ácido acético $45 \%$. Los preparados se hicieron permanentes removiendo el cubreobjetos por congelamiento con nitrógeno líquido y montando con Euparal.

Los cariogramas de cada célula se realizaron a partir de fotomicrografías tomadas en microscopio con contraste de fases Zeiss Axiophot y cámara digital Leica DFC300FX. Las variables calculadas fueron: índice braquial $(\mathrm{r}=1 / \mathrm{s}$ donde $1=$ largo del brazo mayor, $\mathrm{s}=$ largo del brazo menor; Levan et al., 1964), largo total (c) de cada par cromosómico, largo cromosómico promedio (C), largo total del genoma haploide (lt), índice braquial promedio (R), razón entre el cromosoma mayor y el menor del complemento (Rz). Los índices de asimetría se calcularon según Romero Zarco (1986), como sigue:

Índice de asimetría intracromosómica: $\mathrm{A}_{1}=1-\Sigma \mathrm{b} 1$ : b2 / $\mathrm{n}$

siendo $\mathrm{n}=$ número de pares de cromosomas homólogos, b1= largo promedio de brazos cortos en todos los pares de cromosomas homólogos, b2= largo promedio de brazos largos en todos los pares de cromosomas homólogos. Esta ecuación está formulada para obtener valores bajos cuando los cromosomas tienden a ser metacéntricos.

Índice de asimetría intercromosómica: $\mathrm{A}_{2}=\mathrm{s} / \mathrm{x}$ siendo $\mathrm{s}=$ desviación estándar $\mathrm{y} \mathrm{x}=\mathrm{el}$ promedio de largo cromosómico para cada muestra. Este índice refleja la asimetría debida a la variabilidad de las longitudes de todos los cromosomas del complemento.

Se empleó también la clasificación de categorías de asimetría de Stebbins (1971) y los satélites se clasificaron según Battaglia (1955). Los cariogramas se construyeron organizando los cromosomas en grupos de acuerdo a su índice braquial, ordenándolos luego por largo decreciente dentro de cada categoría. Finalmente, se los numeró consecutivamente usando este mismo esquema. Con el programa InfoStat (Di Rienzo et al., 2009) se compararon las variables $\mathrm{C}, \mathrm{lt}, \mathrm{Rz}, \mathrm{R}, \mathrm{A}_{1}$ y $\mathrm{A}_{2}$ con ANAVA y Test de Tukey (Scheffé, 1959; Sokal \& Rohlf, 1982) con el objetivo de determinar si existen diferencias significativas entre las especies.

\section{Resultados}

Las especies de Flourensia estudiadas presentaron $2 \mathrm{n}=36$. En la Fig. 1 se presentan fotomicrografías de células en metafase de cada una y en la Tabla 2 datos obtenidos para las variables analizadas.

Las fórmulas cariotípicas se informan por primera vez y resultaron de interés ya que varían entre las especies. F. campestris, F. leptopoda y F. tortuosa comparten la fórmula con 14 pares $m$ y 4 sm, mientras que $F$. hirta, $F$. niederleinii y $F$. oolepis presentan 12 pares $m$ y $6 \mathrm{sm}$ (Tabla 2, Fig. 2).

Con respecto al tamaño cromosómico, F. hirta y $F$. leptopoda muestran la mayor longitud promedio (2,97 y $2,86 \mu \mathrm{m}$ respectivamente), con diferencias significativas con respecto a los restantes taxones, siendo el menor valor para $F$. campestris $(2,11 \mu \mathrm{m})$. El largo total del genoma haploide es concordante con lo antes citado.

Los índices de asimetría (Tabla 2) muestran que $F$. campestris es la especie más simétrica, mientras que, $F$. niederleinii y $F$. oolepis son las más asimétricas. Cabe aclarar que solo en el índice de asimetría intracromosómica $\mathrm{A}_{1} \mathrm{y}$ en el índice $\mathrm{R}$ se encontraron diferencias estadísticamente significativas entre las especies (Tabla 2). Por último, $F$. hirta y F. niederleinii mostraron mayor índice de asimetría intercromosómica $\mathrm{A}_{2} \mathrm{y}$ mayor índice $\mathrm{Rz}$, aunque no se encontraron diferencias estadísticamente significativas (Tabla 2). La clasificación de Stebbins (1971) resultó similar para todos los taxones analizados: A2. 
Tabla 2. Cariotipos de especies de Flourensia $(2 n=36)$. C: largo cromosómico en micras, entre paréntesis el rango (longitud del cromosoma menor-longitud del cromosoma mayor), It: largo total del genoma haploide en micras, Rz: razón entre las longitudes del cromosoma mayor y el menor del complemento, R: índice braquial, $A_{1}$ : índice de asimetría intracromosómica, $A_{2}$ : índice de asimetría intercromosómica. Todas las variables expresadas como promedio en $\mu \mathrm{m} \pm$ desvío estándar. Anova con $\mathrm{p} \leq 0,05$ y 5 grado de libertad. ${ }^{*}=$ variable con diferencias significativas entre las especies. Diferentes letras muestran diferencias significativas para el Test de Tukey.

\begin{tabular}{|c|c|c|c|c|c|c|c|}
\hline ESPECIE & $\begin{array}{c}\text { Fórmula } \\
\text { cariotípica }\end{array}$ & $\mathrm{C}$ * & It * & $\mathbf{R z}$ & $\mathbf{R} *$ & $A_{1} *$ & $A_{2}$ \\
\hline F. campestris & $14 m+4 s m$ & $\begin{array}{c}2,11 \pm 0,13 \mathrm{c} \\
(2,02-2,34)\end{array}$ & $37,95 \pm 2,35 \mathrm{c}$ & $1,68 \pm 0,16 \mathrm{a}$ & $1,41 \pm 0,06 \mathrm{c}$ & $0,24 \pm 0,03 \mathrm{c}$ & $0,13 \pm 0,02 \mathrm{a}$ \\
\hline F. hirta & $12 m+6 s m$ & $\begin{array}{c}2,97 \pm 0,39 \text { a } \\
(2,56-3,43)\end{array}$ & $53,42 \pm 7,08 \mathrm{a}$ & $1,93 \pm 0,34 \mathrm{a}$ & $1,54 \pm 0,03 \mathrm{ab}$ & $0,3 \pm 0,01 \mathrm{ab}$ & $0,16 \pm 0,03 a$ \\
\hline F. leptopoda & $14 m+4 s m$ & $\begin{array}{c}2,86 \pm 0,14 \text { a } \\
(2,9-3,71)\end{array}$ & $51,5 \pm 2,47 a$ & $1,82 \pm 0,15 \mathrm{a}$ & $1,5 \pm 0,02 \mathrm{ab}$ & $0,3 \pm 0,01 \mathrm{ab}$ & $0,14 \pm 0,02 a$ \\
\hline F. niederleinii & $12 m+6 s m$ & $\begin{array}{c}2,83 \pm 0,24 a b \\
(2,52-3,1)\end{array}$ & $50,95 \pm 4,33 \mathrm{ab}$ & $1,89 \pm 0,15 a$ & $1,59 \pm 0,04 \mathrm{a}$ & $0,33 \pm 0,01 \mathrm{a}$ & $0,16 \pm 0,02 \mathrm{a}$ \\
\hline F. oolepis & $12 m+6 s m$ & $\begin{array}{c}2,69 \pm 0,31 a b \\
(2,41-3,13)\end{array}$ & $48,41 \pm 5,59 a b$ & $1,79 \pm 0,21 \mathrm{a}$ & $1,59 \pm 0,04 \mathrm{a}$ & $0,32 \pm 0,01 \mathrm{a}$ & $0,14 \pm 0,01 \mathrm{a}$ \\
\hline F. tortuosa & $14 m+4 s m$ & $\begin{array}{c}2,33 \pm 0,13 b c \\
(1,63-2,86)\end{array}$ & $41,92 \pm 2,27 b c$ & $1,78 \pm 0,07 \mathrm{a}$ & $1,47 \pm 0,07 \mathrm{bc}$ & $0,27 \pm 0,04 \mathrm{bc}$ & $0,14 \pm 0,02 \mathrm{a}$ \\
\hline
\end{tabular}

Con respecto a la presencia de satélites, $F$. campestris es la única especie en la que no se visualizaron, en tanto que para las restantes varían su número y su ubicación (Fig. 1, 2). F. leptopoda, $F$. oolepis y $F$. tortuosa tienen un par mientras que $F$. niederleinii y $F$. hirta tienen dos pares. Todos son microsatélites con $0,3 \mu \mathrm{m}$ de longitud en promedio y están ubicados en una posición terminal en un brazo corto (Fig. 1, 2). Su visualización es variable: en un $65-75 \%$ de las células de todas las especies no se han observado.

\section{Discusıón}

Las especies de Flourensia estudiadas presentaron $2 \mathrm{n}=36$, lo cual concuerda con lo informado por otros autores en material de otras procedencias (Dillon, 1979; Di Fulvio, 1977; Wulff, 1996). Asteraceae presenta un gran rango de números cromosómicos, desde $2 \mathrm{n}=4$ hasta $2 \mathrm{n}=$ ca. 432 , siendo el mas frecuente $2 \mathrm{n}=18$. El número básico también es variable, entre $\mathrm{x}=2 \mathrm{y}$ $\mathrm{x}=11, \mathrm{y}$, además, hay gran cantidad de números básicos secundariamente derivados. Sumado a esto, el 58,3\% de los géneros son poliploides, con niveles hasta $48 x$. Pese a esta gran heterogeneidad, se considera que el número básico ancestral de la familia es $x=9$ (Semple \& Watanabe, 2009).

Con respecto a la Alianza Heliantheae, existen al menos dos posturas sobre el posible número básico. Baldwin et al. (2002) proponen a $\mathrm{x}_{2}=18$ como ancestral, en tanto que Semple \& Watanabe (2009) a $x_{2}=19$ por encontrarse en la mayoría de las tribus de la Alianza, incluyendo Heliantheae; estos últimos autores señalan que $\mathrm{x}_{2}=18,17,15$ y 11 serían derivados por disploidía decreciente. Heliantheae presenta números cromosómicos que van desde $\mathrm{n}=3$ hasta ca. 50 , con mayor frecuencia de $\mathrm{n}=17-19$ (Robinson et al., 1981).

En cuanto al origen del número cromosómico de Flourensia $(2 \mathrm{n}=36)$, Wulff (1996) postuló que serían diploides $(\mathrm{x}=18)$, pero plantea que, teniendo en cuenta el número básico de la familia $(x=9)$, se trataría de tetraploides. En contraste, si aceptamos que el número básico de la Alianza y de la tribu es $\mathrm{x}_{2}=19$, el género habría sufrido un evento de disploidía decreciente, el cual disminuyó el número $\mathrm{x}_{2}=19$ a x $_{2}=18$.

De los géneros relacionados a Flourensia, Geraea también presenta $\mathrm{n}=18$, Encelia y Enceliopsis $\mathrm{n}=17-18$ y Helianthella $\mathrm{n}=15$ (Robinson et al., 1981; Dillon, 1984, Clark, 1998), aunque no hay estudios cariotípicos comparativos. 


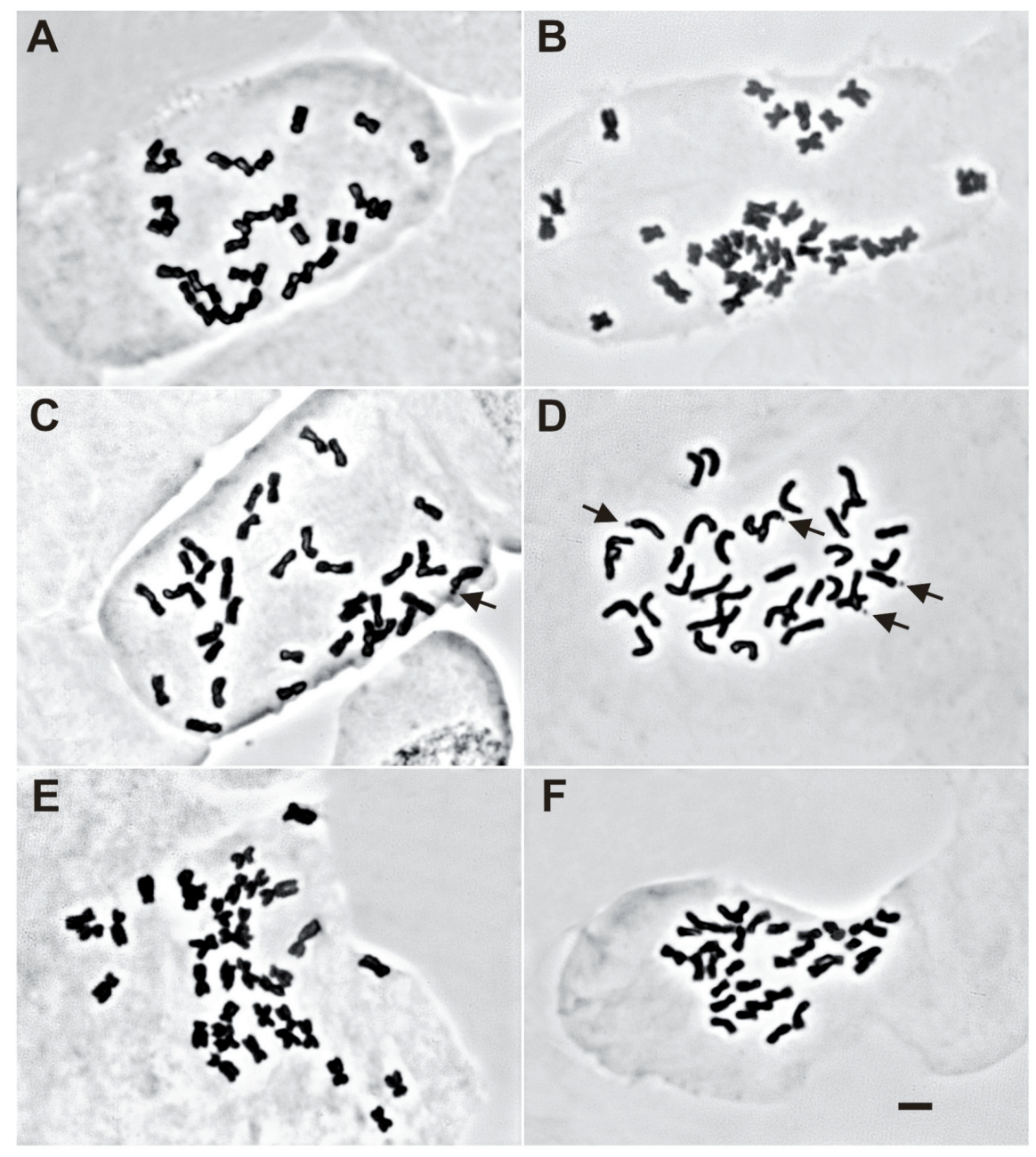

Fig. 1. Metafases mitóticas de especies de Flourensia. A: F. campestris. B: F. hirta. C: F. leptopoda. D: F. niederleinii. E: F. oolepis. F: F. tortuosa. Flechas indican satélites. Escala $=3 \mu \mathrm{m}$, todas a la misma escala.

Dentro de la tribu, Helianthus ha sido el más estudiado citológicamente, por su importancia económica. La mayoría de las especies, incluida $H$. annuus L., presenta $2 \mathrm{n}=2 \mathrm{x}=34$ con 4 pares st y 13 $m$ a $s m$ (Schrader et al., 1997; Vanzela et al., 2002). La poliploidía fue muy importante en su evolución cariotípica, existiendo tetraploides y hexaploides con similares posiciones del centrómero (Vanzela et al., 2002). Por su parte, en Flourensia no habrían ocurrido eventos de poliploidía.

En Eupatorieae, otra tribu de la Alianza, el número cromosómico $\mathrm{n}=18$ aparece en unas pocas subtribus basales, filogenéticamente cercanas a Heliantheae; en contraste, la mayor cantidad de especies presenta $\mathrm{n}=10$, seguida en importancia por $\mathrm{n}=17$. Este último sería el número básico de la tribu y la evolución del número cromosómico habría avanzado en una dirección descendente (Watanabe et al., 1995).

Tanto en Eupatoriaeae (Watanabe et al., 1995), como en Heliantheae (Baldwin, 2009), se ha observado una relación entre la cantidad de cromosomas y el hábito de los taxones. De modo que la presencia de números cromosómicos altos se ha relacionado con hábitos arbustivos y arborescentes que habitan ambientes estables. Por el contrario, la reducción en el número cromosómico se asocia con un hábito herbáceo, en plantas anuales o bianuales, 
Bol. Soc. Argent. Bot. 49 (2) 2014
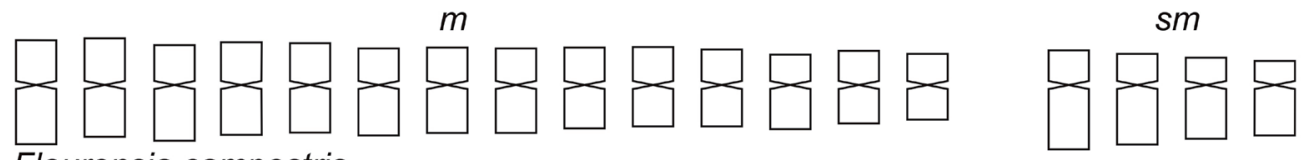

Flourensia campestris

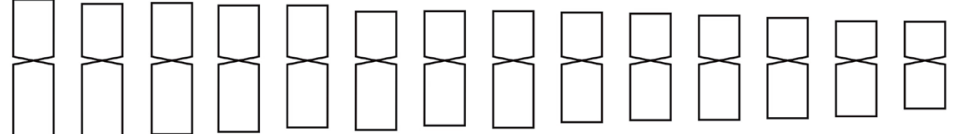

F. leptopoda

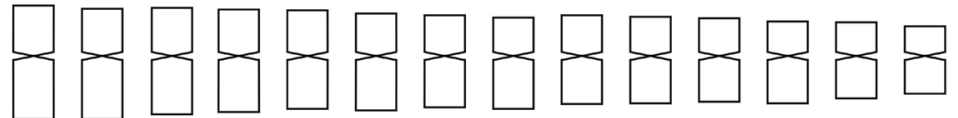

\section{F. tortuosa}
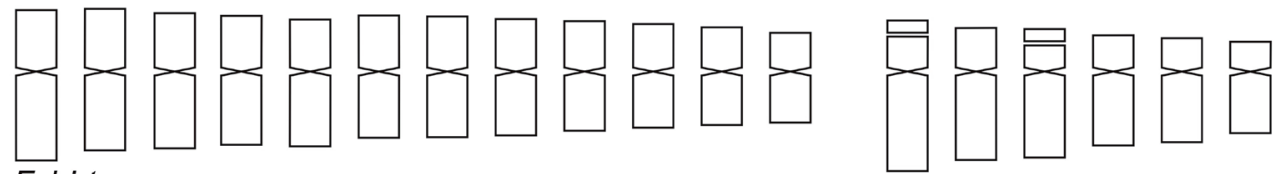

F. hirta

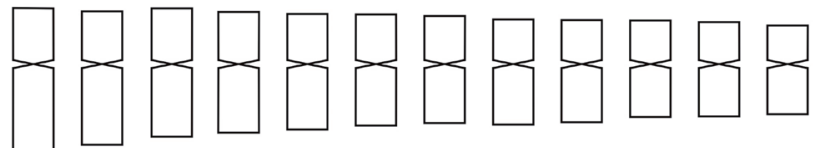

F. niederleinii

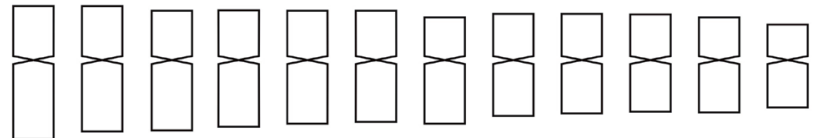

F. oolepis
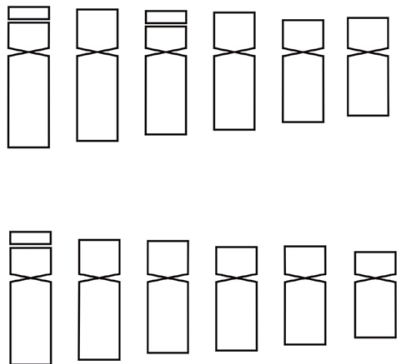

$3 \mu \mathrm{m}$

Fig. 2. Idiogramas de especies de Flourensia basado en los valores medios. $m$ : metacéntico, sm: submetacéntrico. Escala $=3 \mu \mathrm{m}$, todas a la misma escala.

lo cual podría fomentar la explotación de hábitats con recursos efímeros. En este sentido, las especies de Flourensia seguirían este patrón general, ya que son perennes y con elevado número cromosómico.

Los datos obtenidos tienen valor taxonómico ya que se han encontrado diferencias entre casi todas las especies. Según las fórmulas cariotípicas, es posible discriminar dos grupos, los cuales difirieron por la cantidad de cromosomas $\mathrm{sm}$. Por otro lado, se encontraron diferencias estadísticas en la mayoría de las variables analizadas, excepto $\mathrm{Rz}$ y $\mathrm{A}_{2}$. Así, $F$. campestris se diferenció de las demás especies por presentar los menores valores en longitud cromosómica promedio y largo total del genoma haploide; asimismo, fue la especie más simétrica y no presentó satélites. En contraste, las especies que resultaron con mayores valores de longitud son $F$. hirta y F. leptopoda, mientras que $F$. niederleiini y $F$. oolepis fueron las más asimétricas.

Estas diferencias se corresponden, en parte, con los datos de distribución geográfica (Ariza Espinar, 2000), así como también con los referidos a exomorfología y anatomía de órganos vegetativos, 
reportados con anterioridad para las mismas especies (Delbón et al., 2007a, b, 2012). F. hirta, F. leptopoda y $F$. niederleinii presentan una distribución acotada que se restringe a pequeñas poblaciones en las Provincias de La Rioja, Catamarca y San Juan (Ariza Espinar, 2000). Desde el punto de vista morfoanatómico son consideradas mejor adaptadas al ambiente xérico en que habitan, ya que tienen menor tamaño foliar, reducción en la cantidad de hacecillos foliares y mayor frecuencia estomática, entre otras características (Delbón et al., 2012). En concordancia, estas especies son las que presentaron mayor largo cromosómico promedio. F. campestris, F. oolepis y $F$. tortuosa son las especies consideradas menos adaptadas al ambiente xérico (Delbón et al., 2007a, b), lo cual se puede relacionar con su amplia distribución geográfica y su mayor abundancia (Ariza Espinar, 2000); estas especies son, además, las que presentaron menor largo cromosómico.

\section{Agradecimientos}

A la Secretaría de Ciencia y Técnica de la Universidad Nacional de Córdoba (SECYT) por financiamiento. ND es becaria de CONICET y estudiante de Doctorado en Ciencias Biológicas de la Universidad Nacional de Córdoba. GB es miembro de la carrera del investigador de CONICET.

\section{Bibliografía}

ARIZA ESPINAR, L. 2000. Familia Asteraceae. Tribu Heliantheae. Pródromo de la Flora Fanerogámica de Argentina Central 2: 1-111. Museo Botánico, Córdoba.

BALDWIN, B. 2009. Heliantheae alliance. In: FUNK, V. A., A. SUSANNA, T. F. STUESSY \& R. J. BAYER (eds.), Systematics, Evolution, and Biogeography of Compositae, pp. 689-711. Sheridan Books, Inc., Ann Arbor, Michigan.

BALDWIN, B., B. WESSA \& J. PANERO. 2002. Nuclear rDNA evidence for major lineages of Helenioid Heliantheae (Compositae). Syst. Bot. 27: 161-198.

BARBOZA, G., J. CANTERO, C. NUÑEZ \& L. ARIZA ESPINAR. 2006. Flora medicinal de la provincia de Córdoba (Argentina). Museo Botánico, Córdoba.
BATTAGLIA, E. 1955. Chromosome morphology and terminology. Caryologia 8: 179-187.

CARR, G., R. KING, A. POWELL \& H. ROBINSON. 1999. Chromosome numbers in Compositae. XVIII. Amer. J. Bot. 86: 1003-1013.

CLARK, C. 1998. Phylogeny and adaptation in the Encelia Alliance (Asteraceae, Heliantheae). Aliso 17: 89-98.

DELBÓN, N., M. T. COSA \& G. BERNARDELLO. 2012. Exomorfología y anatomía de órganos vegetativos aéreos en especies de Flourensia DC. (Asteraceae) con importancia fotoquímica. Acta Bot. Brasíl. 26: $2-10$.

DELBÓN, N., M. T. COSA \& N. DOTTORI. 2007a. Anatomía de órganos vegetativos en Flourensia campestris y F. oolepis (Asteraceae), con especial referencia a las estructuras secretoras. Arnaldoa 14: 61-70.

DELBÓN, N., M. T. COSA, N. DOTTORI \& L. STIEFKENS. 2007b. Estudio de la epidermis foliar en Flourensia campestris y F. oolepis (Asteraceae). Bol. Soc. Argent. Bot. 42: 245-250.

Di FULVIO, T. E. 1977. Recuentos cromosómicos en Angiospermas Argentinas. III. Kurtziana 10: 69-72.

DILLON, M. O. 1979. In: LOVE, A. (ed.), IOPB chromosome number reports. LXIII. Taxon 28: 278279.

DILLON, M. O. 1981. Three new species of Flourensia (Asteraceae-Heliantheae) from South America. Ann. Missouri Bot. Gard. 68: 105-111.

DILLON, M. O. 1984. A systematic study of Flourensia (Asteraceae, Heliantheae). Fieldiana Bot. 16: 1-67.

DILLON, M. O. 1986. A new species of Flourensia (Asteraceae: Heliantheae) from northern Peru. Brittonia 38: 32-34.

DI RIENZO, J., F. CASANOVES, M. BALZARINI, L. GONZALEZ, M. TABLADA \& C. ROBLEDO. InfoStat, versión 2009. Grupo InfoStat, F. C. A. U.N.C., Córdoba.

FEHLBERG, S. D. \& T. A. RANKER. 2007. Phylogeny and biogeography of Encelia (Asteraceae) in the Sonoran and peninsular deserts of based on multiple DNA sequences. Syst. Bot. 31: 692-699.

FUNK, A., A. SUSANNA, T. STUESSY \& H. ROBINSON. 2009. Classification of Compositae. In: FUNK, V. A., A. SUSANNA, T. F. STUESSY \& R. J. BAYER (eds.), Systematics, Evolution, and Biogeography of Compositae, pp. 171-192. Sheridan Books, Ann Arbor.

JONG, J. 1997. Laboratory manual of plant cytological techniques. Royal Bot. Garden, Edinburgh.

JORAY, M., M. DE ROLLAN, G. RUIZ, S. PALACIOS \& M. CARPINELLA. 2011. Antibacterial Activity of Extracts from Plants of Central Argentina. Isolation of an Active Principle from Achyrocline satureioides. Planta Med. 77: 95-100. 
KATINAS, L., D. GUTIERREZ, M. GROSSI \& J. CRISCI. 2007. Panorama de la familia Asteraceae (=Compositae) en la República Argentina. Bol. Soc. Argent. Bot. 42: 113-129.

MAYER, J. R. 1945. Prefixing with paradiclorobemzene to facilite chromosome study. Stain Technol. 20: 121-125.

MOLAU, U. 1986. In: DILLON, M. O. 1986. A new species of Flourensia (Asteraceae: Heliantheae) from northern Peru. Brittonia 38: 32-34.

MORENO, N., L. STIEFKENS, M. L. LAS PEÑAS, A. BARTOLI, R. TORTOSA \& G. BERNARDELLO. 2012. Molecular cytogenetic studies of the "Xanthocephalum group" (Asteraceae). Plant Syst. Evol. 298:1503-1514.

LEVAN, A., K. FREDGA \& A. SANDBERG. 1964. Nomenclature for centomeric position on chromosomes. Hereditas 52: 201-220.

PANERO, J. L. 2005. New combinations and infrafamilial taxa in the Asteraceae. Phytologia 87: 1-14.

PANERO, J. L. 2007a. Key to the tribes of the Heliantheae Alliance. In: KADEREIT, J. W. \& C. JEFFREY (eds.), Families and Genera of Vascular Plants, vol. VIII, Flowering Plants, Eudicots, Asterales, pp. 391395. Springer-Verlag, Berlin.

PANERO, J. L. 2007b. Compositae: tribe Heliantheae. In: KADEREIT, J. W. \& C. JEFFREY (eds.), Families and Genera of Vascular Plants, vol. VIII, Flowering Plants, Eudicots, Asterales, pp. 440-477. SpringerVerlag, Berlin.

PANERO, J. L., R. K. JANSEN \& J. A. CLEVINGER. 1999. Phylogenetic relationships of subtribe Ecliptinae (Asteraceae: Heliantheae) based on chloroplast DNA restriction site data. Amer. J. Bot. 86: 413-427.

POWELL, A. M. 1984. In: DILLON, M. O. A systematic study of Flourensia (Asteraceae, Heliantheae). Fieldiana Bot. 16: 1-67.

ROBINSON, H., A. POWELL, R. KING \& J. WEEDIN 1981. Chromosome numbers in Compositae XII: Heliantheae. Smithsonian Contrib. Bot. 52: 1-28.

ROMERO ZARCO, C. 1986. A new method for estimating karyotype asymmetry. Taxon 35: 526230.

SCHEFFE, H. 1959. The Analisis of Variance. John Wiley, New York.

SCHRADER, O., R. AHNE, J. FUCHUS \& I. SCHUBERT. 1997. Karyotype analysis of Helianthus annuus using Giemsa banding and fluorescence in situ hybridization. Chrom. Res. 5: 451-456.

SEMPLE, J. C \& K. WATANABE. 2009. A review of chromosome numbers in Asteraceae with hypotheses on chromosomal base number evolution. In: FUNK, V. A., A. SUSANNA, T. F. STUESSY \& R. J. BAYER (eds.), Systematics, Evolution, and
Biogeography of Compositae, pp. 61-72. Sheridan Books, Ann Arbor.

SHAN F., G. Yan \& J. A. Plummer. 2003. Karyotype evolution in the genus Boronia (Rutaceae). Bot. J. Linn. Soc. 142: 309-320.

SIMPSON, B. 2009. Economic importance of Compositae. In: FUNK, V. A., A. SUSANNA, T. F. STUESSY \& R. J. BAYER (eds.), Systematics, Evolution, and Biogeography of Compositae, pp. 45-58. Sheridan Books, Ann Arbor.

SOKAL, R. \& F. ROHLF. 1981. The Principles and Pratice of Statistic in Biological Research. Freeman and Co., San Francisco.

STEBBINS, G. L. 1971. Chromosomal evolution in higher plants. E. Arnold, London.

STROTHER, J. L. 1983. More chromosome studies in Compositae. Amer. J. Bot. 70: 1217-1224.

TRILLO, C., P. DEMAIO, S. COLANTONIO \& L. GALETTO. 2007. Conocimiento actual de plantas tintóreas por los pobladores del valle de Guasapampa, provincia de Córdoba. Kurtziana 33: 65-71.

TURNER, B. L. \& M. JOHNSTON. 1961. Chromosome numbers in the Compositae. III. Certain Mexican species. Brittonia 13: 64-69.

URDAMPILlETA, J. D., M. S. FERRUCCI \& A. VANZELA. 2005. Karyotype differentiation between Koelreuteria bipinnata and K. elegans ssp. formosana (Sapindaceae) based on chromosome banding patterns. Bot. J. Linn. Soc. 149: 451-455.

URIBURU, M., J. DE LA FUENTE, J. PALERMO, R. GIL \& V. SOSA. 2004. Constituents of two Flourensia species. Phytochemistry 65: 2039-2043.

VACA RUIZ, M. L., A. LACIAR, O. DONADEL, J. SAAD \& R. CARRIZO FLORES. 2006. Antilisterial activity of plant essential oils from western region of Argentina. Ann. Microbiol. 56: 369-371.

VANZELA, A., C. RUAS, M. OLIVEIRA \& P. RUAS. 2002. Characterization of diploid, tetraploid and hexaploid Helianthus species by chromosome banding and FISH with 45S rDNA probe. Genetica 114: 105-111.

WAISMAN, C. E., E. ROZENBLUM \& J. HUNZIKER. 1986. Estudios cariológicos en Compositae. III. Darwiniana 27: 179-189.

WATANABE, K., R. KING, T. YAHARA, M. ITO, J. YOKOYAMA, T. SUZUKI \& D. CRAWFORD. 1995. Chromosomal, Cytology and Evolution in Eupatorieae (Asteraceae). Ann. Missouri Bot. Gard. 82: 581-592.

WEEDIN, J. F. \& A. M. POWELL. 1980. In: LOVE, A. (ed.), IOPB chromosome number reports. LXIX. Taxon 29: 716-718.

WEISS-SCHNEEWEISS, H., T. F. STUESSY \& J. L. VILLASEÑOR. 2009. Chromosome numbers, karyotypes and evolution in Melapodium 


\section{N. Delbón et al. - Estudios cariotípicos en Flourensia}

(Asteraceae). Int. J. Plant Sci. 170: 1168-1182.

WULFF, A. F., J. H. HUNZIKER \& A. ESCOBAR. 1996. Estudios cariológicos en Compositae. VII. Darwiniana 34: 213-231.

ZARDINI, E. 1984. Etnobotánica de Compuestas
Argentinas con Especial Referencia a su uso Farmacológico. Acta Farmacol. Bonaerense 3: 77-99.

Recibido el 21 de octubre de 2013, aceptado el 25 de marzo de 2014. 
